\title{
Treatment of maxillary molar with furcation involvement: Case report
}

\section{Correia F', Costa A}

\section{Affiliations:}

1. Department of Oral Surgery and Periodontology, Faculty of Dental Medicine, University of Porto, Porto, Portugal

\section{Corresponding Author:}

Francisco Correia franciscodcorreia@gmail.com

\begin{abstract}
Purposes: The aim of this clinical report is to discuss the advantages and the steps of the root separation and resection approach in a maxillary molar with a class III furcation of the palatine root with a follow-up of 24 months. Case report: A patient with tooth mobility and bleeding after probing, was diagnosed with a Chronic Periodontitis Generalized Moderate and Localized Severe.

Hygienic phase with oral hygiene instructions, scaling and root planning was performed. Due to class III furcation of the palatine root of the teeth 16 and some remaining pockets deepths, was decided to execute an apical repositioning flap on the $1^{\text {st }}$ sextant among with the palatine root amputation combined with the endodontic treatment. During the surgical procedure, it was also performed a reduction of the palate thickness from teeth 15 to 17 ; in distal area of the 17 a distal wedged was performed in order to remove all the pockets. After the surgery, the patient was inserted in a long-lasting individualized supportive periodontal treatment. This case has a follow up of 24 months.

Conclusions: Similar survival rates are described in the literature when we compared the root amputation with the dental implants survival rate, but the first one is cheaper and allows to keep the natural tooth. We can't forget that the periodontitis is a risk factor of peri-implantitis.

This clinical case shows that, with a high motivated patient and with the complete periodontal treatment, is possible to obtain excellent results with root separation and resection in maxillary molars class III.
\end{abstract}

\section{KEYWORDS}

Periodontal treatment, resective therapy, furcation lesion, multirooted teeth, case reports. 


\section{INTRODUCTION}

Periodontal pathology is a multifactorial disease which primary etiologic factor are specific bacteria. A high variability of the severity of the disease among individuals is influenced by factors that can be systemic, genetic, behavioural or environmental. ${ }^{1}$

The loss of insertion (destruction of bone and connective tissue) around molars (or/and multirradicular premolars) can affect the furcation area, due to the complex anatomy of this area. The periodontal treatments of multi rooted teeth with furcation involvement are technical sensitive.

Molars with furcation involvement represent a major challenge in periodontal treatment., 3

The aim of the treatment of furcation's defects in multirradicular teeth are two: ${ }^{4}$

1. Remove the bacterial plaque of the exposed surface of the root complex

2. Restore the healthy anatomy of the affected surfaces in order to promote an adequate plaque control.

According to the severity of the furcation defect, ${ }^{5}$ different treatment approaches are recommended. ${ }^{6}$

Grade I - scaling and root planning; furcation plasty

Grade II - furcation plasty, tunnel preparation, RSR (root separation and resection), GTR (Guided tissue regeneration), teeth extraction

Grade III - tunnel preparation, RSR, teeth extraction

RSR is a frequent option in molars with deepth degree II and III, however, the success of this approach depends of multi criteria: ${ }^{6}$

1. The length of the root trunk (short root trunk is better)

2. Divergence between the root cones (short divergence is more difficult)

3. Length and shape of the root cones (short and small have more are more subjected to mobility after resection 4. Amount of remaining support around individual roots 5. Stability of individual roots

6. Access for oral hygiene

The aim of this clinical case is to discuss the advantages and the steps of the RSR approach in a maxillary molar with a class III furcation of the palatine root with a follow-up of 24 months.

\section{CLINICAL CASE DESCRIPTION}

A healthy (ASA type 1), 60-year-old women patient, non-smoker, with tooth mobility and bleeding after probing. The orthopantomography radiography showed generalized horizontal bone loss around the tooth (Figure 1).

After a complete periodontal evaluation, was diagnosed a Chronic Periodontitis Generalized Moderate and Localized Severe7, during this evaluation session was given the oral hygiene instructions (brush technique and explanation of the importance of the use of interdental brush).

After the first session, scaling and root planning was performed in two different sessions with a week of interval between the two sessions.

Two months after the initial phase, a periodontal evaluation was performed and was decided to execute an apical repositioning flap of the first sextant among with the palatine root amputation of the tooth 16 combined with the endodontic treatment due to class III furcation of the palatine root.

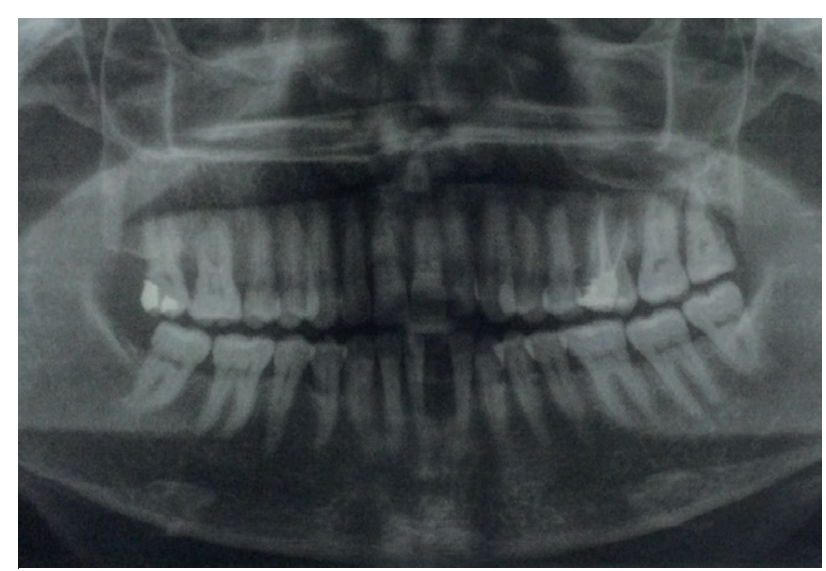

Figure 1. Initial orthopantomography 


\section{SURGICAL PROCEDURE}

Local anaesthetic, articaine (artinibsa ${ }^{\circledR}$ 4\% 1:100.000) was administered for pain and haemorrhagic control and the patient rinsed with $0.12 \%$ chlorhexidine for 1 minute prior the surgery.

An intrasulcular incision was performed in the vestibular side and in the palatine area. In combination with the intrasulcular incision, it was performed a reduction of the palate thickness from teeth 15 to 17; in distal area of the 17 a distal wedged was performed in order to remove all the pockets (Figures 3, 4).

After the incision, a flap was raised and all the granulation tissue and remaining tartar was removed with curettes, perio-set and scaler (Figures 5, 6).

The amputation of the palatal root of the tooth 16 was performed with a cylindrical diamond drill and any remaining granulation tissue still present were cleaned (Figures 7-9). Three horizontal mattress sutures were performed in order to close the flap (Figure 10).

After surgery, was prescribed a rinse with $0.12 \%$ chlorhexidine (eludril perio ${ }^{\circledR}$ ) twice a day for 15 days, 1 pill, 12/ 12 hours for 5 day of Ibuprofen $600 \mathrm{mg}$ (brufen ${ }^{\circledR} 600 \mathrm{mg}$ ) and amoxicillin $1 \mathrm{~g}\left(\right.$ Clamoxyl $\left.^{\circledR} 1 \mathrm{~g}\right) 1$ pill of 12/ 12 hours 1 week. After 15 days, the suture was removed and no complication occurred (Figure 11).
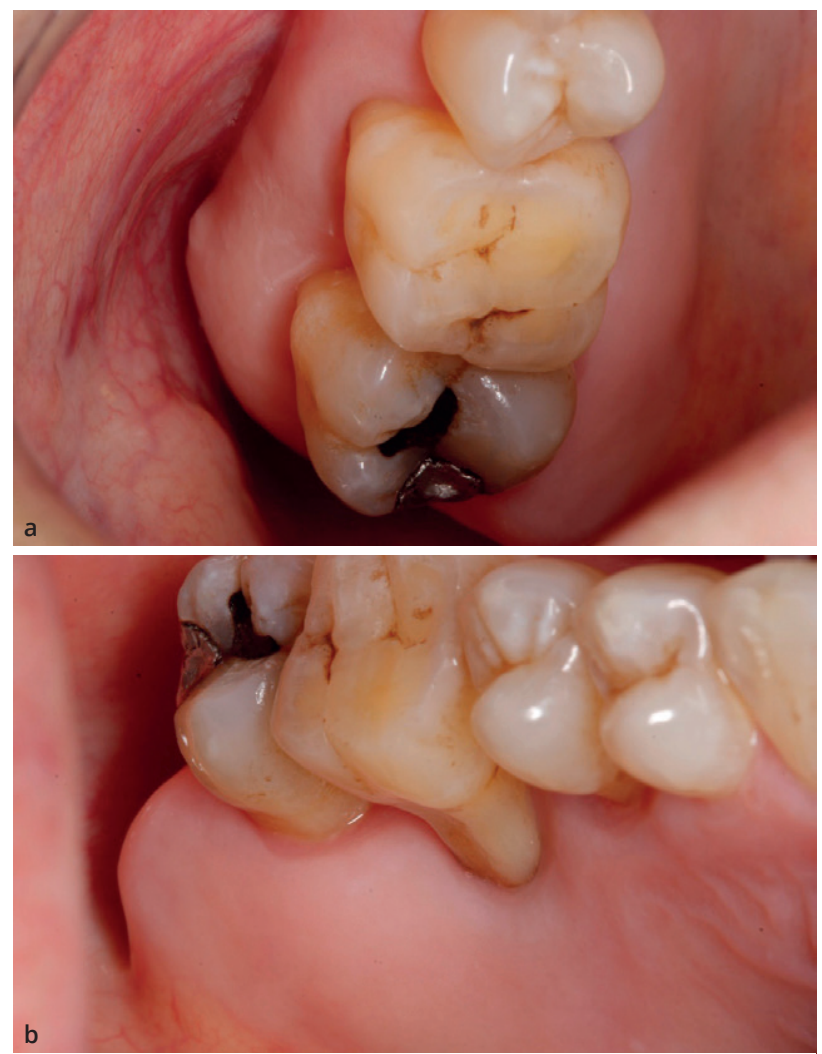

Figures 2a, 2b. Initial images before surgery

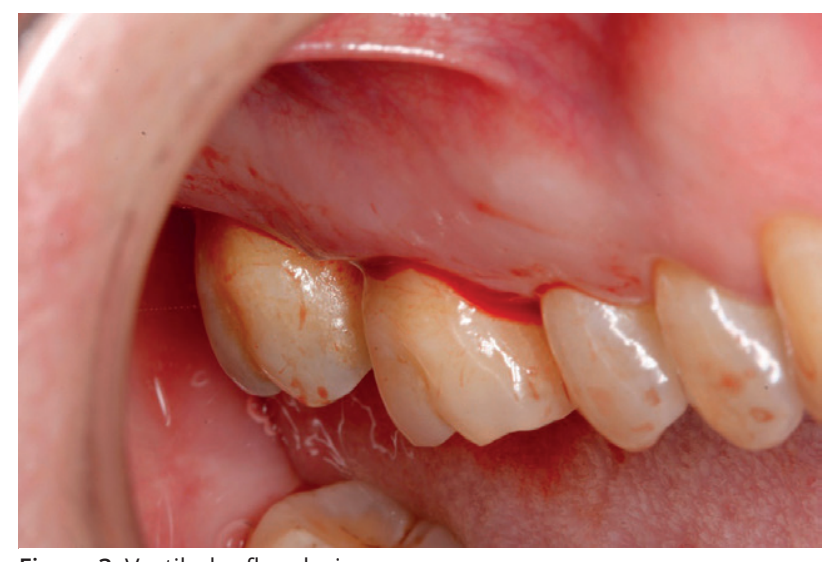

Figure 3. Vestibular flap design

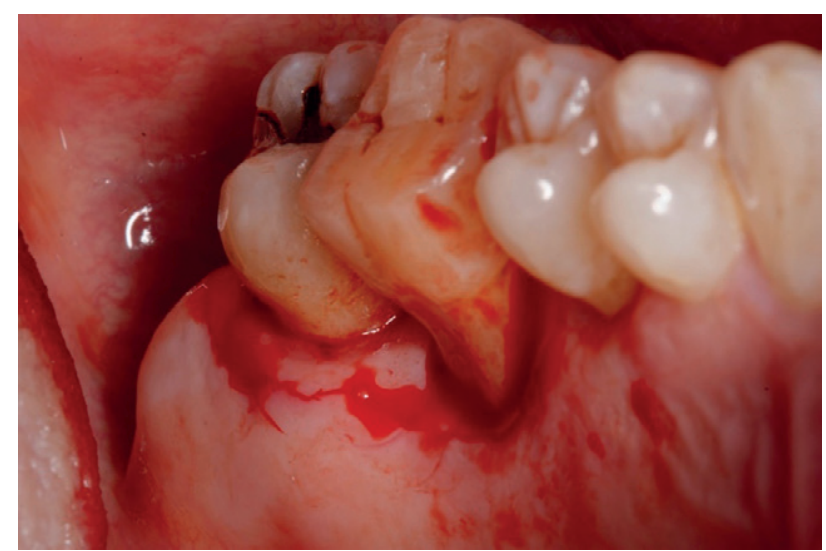

Figure 4. Palatal flap design

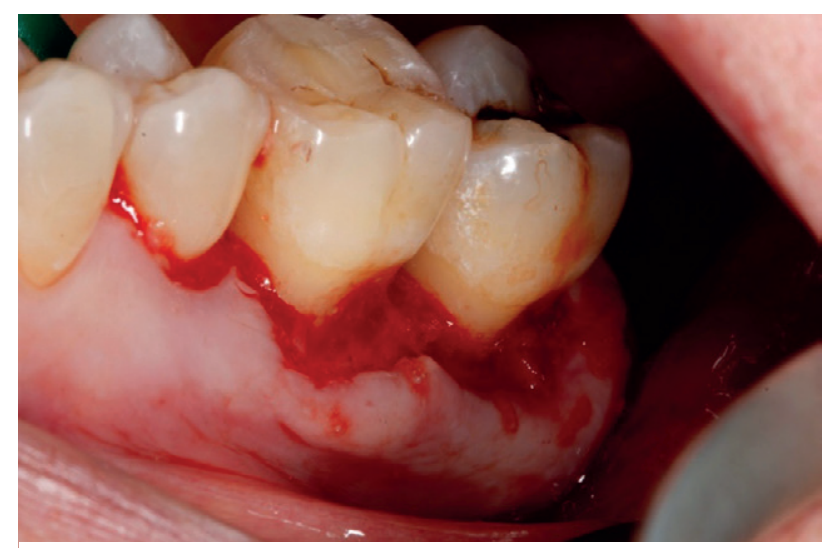

Figure 5. Vestibular flap raised

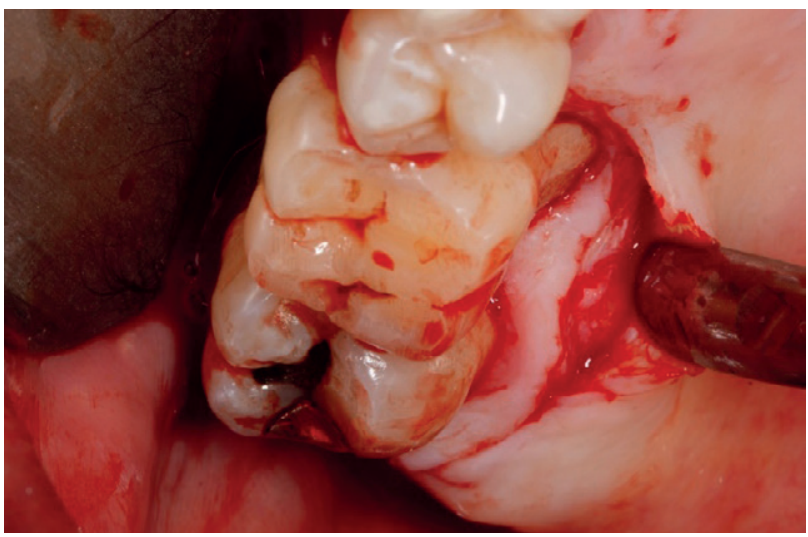

Figure 6. Palatal flap raised 


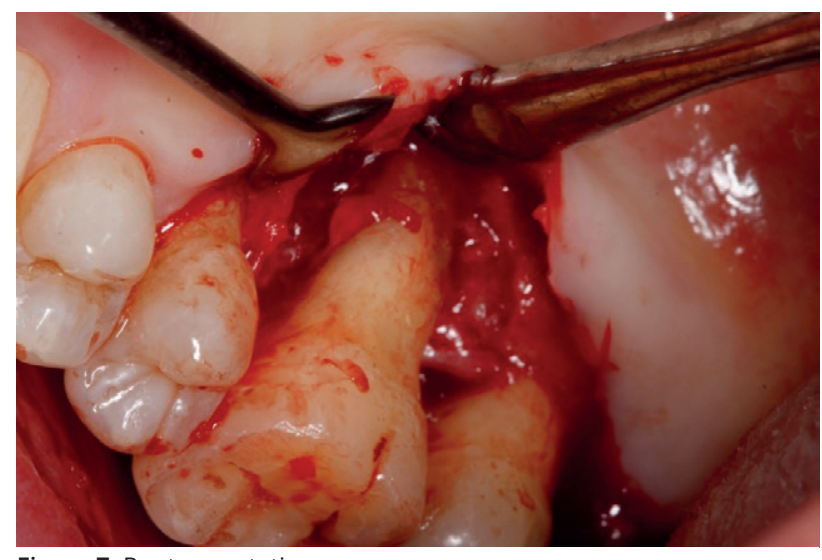

Figure 7. Root amputation sequence

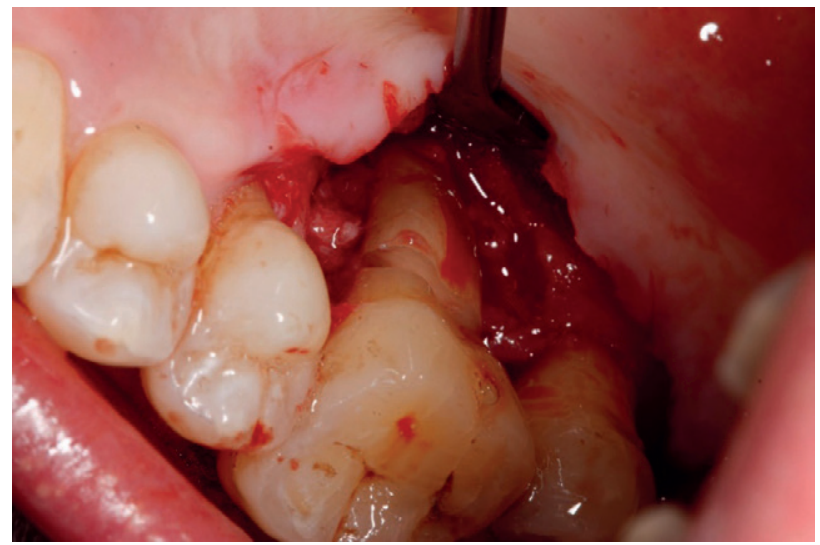

Figure 8. Root amputation sequence

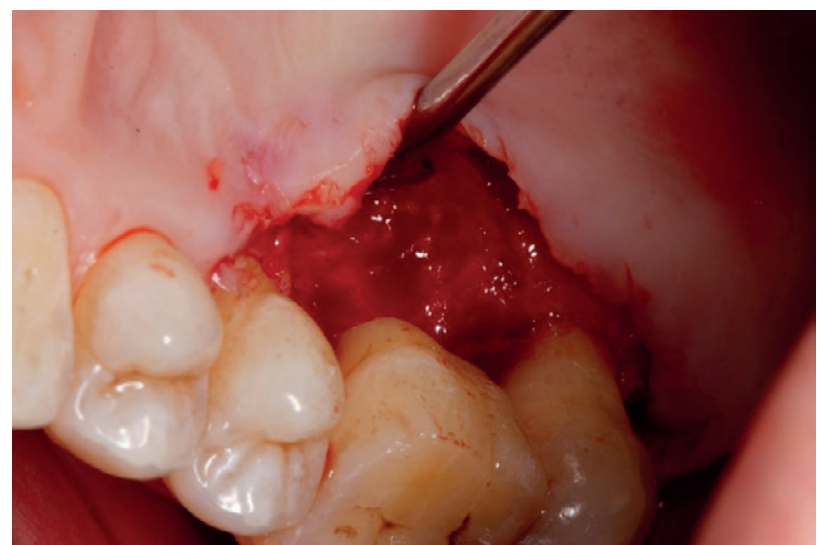

Figure 9. Root amputation sequence

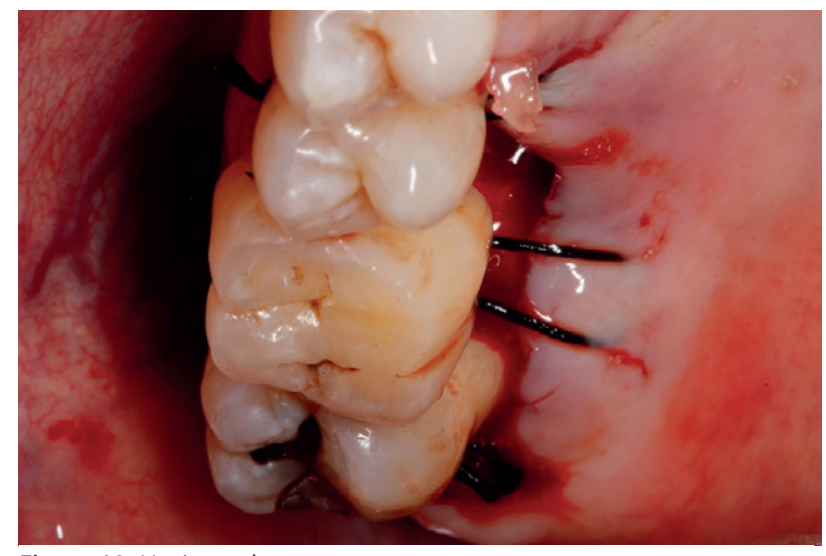

Figure 10. Horizontal mattress suture
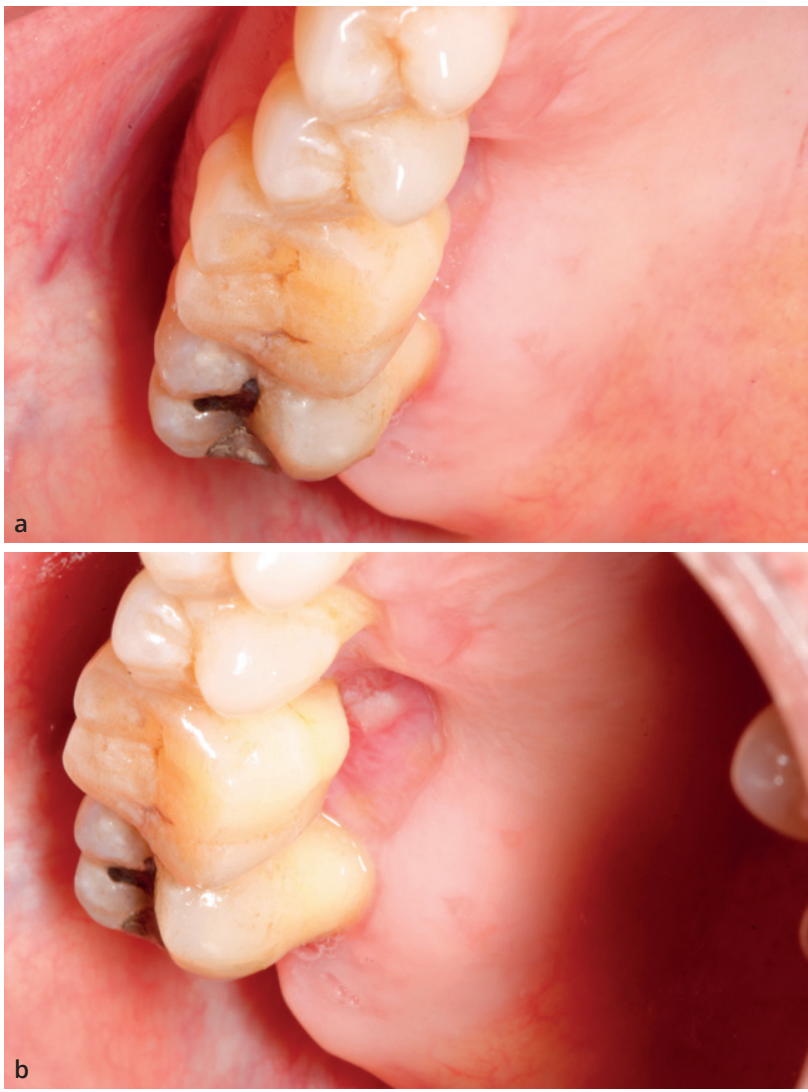

Figure 11a, 11b. 15 days after surgery 


\section{SUPPORTIVE PERIODONTAL TREATMENT}

After the surgery, the patient was included in an individualized supportive periodontal treatment, every three months during the first year and since then, every 4 months. This long-lasting supportive periodontal treatment was feasible because the patient is able to maintain an excellent plaque control and does not have any pocket depth or bedding on probing.

This case has a follow up of 24 months (Figures 12,13).

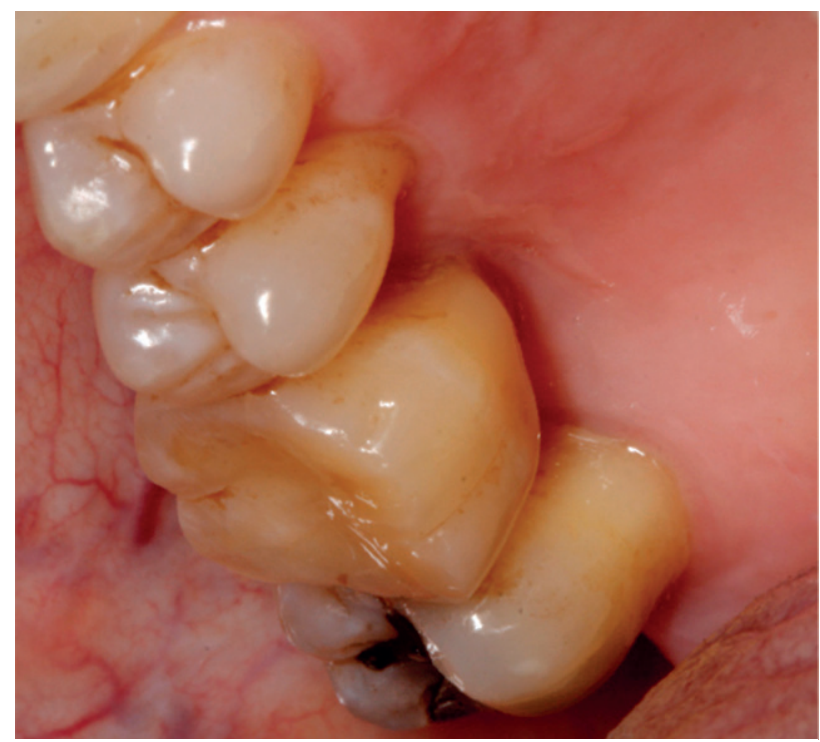

Figure 12. 24 months follow-up: palatal view

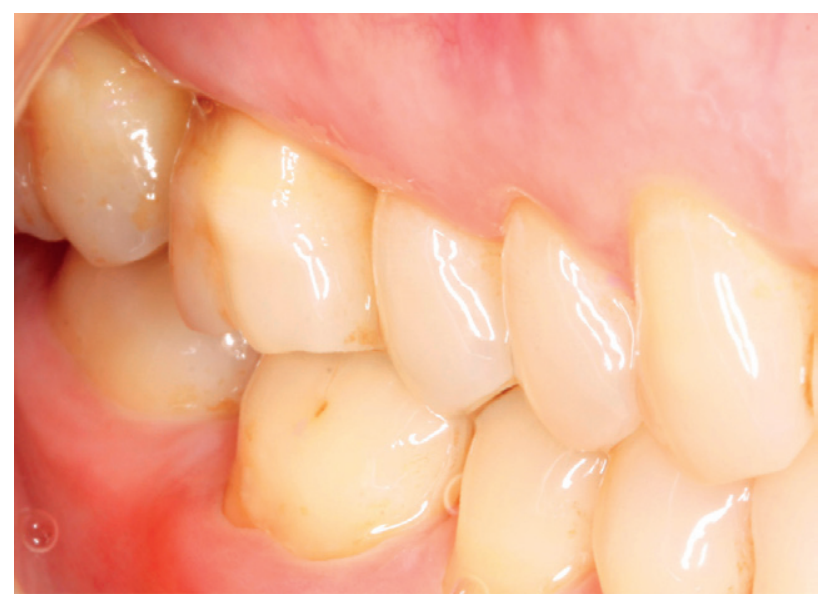

Figure 13. 24 months follow-up: vestibular view

\section{DISCUSSION}

There has been a growing concern among the dental community to maintain the natural teeth, at the same time, there has been a raising awareness among the population about the importance of having regular consultations for this purpose.

The advanced periodontal disease with deep periodontal pockets $(+6 \mathrm{~mm})$ affects 10 to $15 \%$ of the world population, ${ }^{8}$ the periodontal treatment has a very strong scientific base and the treatments have high success rates. ${ }^{9}$

The periodontal treatment in furcated teeth presents a great challenge since there are many factors that determine its success: anatomical factors (dental anatomy), clinical factors (severity of the furcation grade I, II or III lesion), and technical factors (professional experience, difficulty of access). ${ }^{10}$

Simultaneously, the scientific knowledge and alternatives to the rehabilitation with dental implant have increased with high success and survival rates. ${ }^{11}$ This leads us to question our self how far the limits of periodontal treatment should go and when the best alternative is extraction and possible rehabilitation with dental implant and which is the price of these two options. ${ }^{12,13}$

The scientific evidences show that with proper periodontal treatment is possible to maintain teeth; the extraction is performed when, even with a periodontal treatment, is impossible to maintain the tooth due an unfavourable anatomy or lack of proper oral hygiene or when maintaining the tooth does not prove to be the best option in the global treatment. ${ }^{14,15}$

The prognosis of treatment of multi-rooted teeth, is usually worse than single-rooted teeth and additionally, furcation degree III is associated with significantly increased rates of tooth loss. ${ }^{16}$

A lesion in the inter-radicular area of a multirradicular tooth may be associated with problems of the root canal or it may result of occlusal overload; the treatment of teeth with furcation involvement should not be started until an adequate differential diagnosis of the lesion. ${ }^{4}$

When root resection is the clinical option to treat upper molar furcation lesions, the anatomy of the teeth should be well considered, the morphology of each of the roots and their surface area should be evaluated; since these teeth has three root cones, one or two can remain functional after treatment. One of the difficulties in deciding the best therapeutic option for furcation lesions in upper molars, is the fact that the root hemisectomy has a very variable success rate, between 68 and $96.8 \% .^{10}$

The main problem in root separation and resection (RSR) is that the success not depends only of a successful periodontal therapy but also of the success of the endodontic treatment, the restorative procedures and the motivation of the patient. ${ }^{17}$

In a recent study of Derks et al (2017) a retrospective up to 30 years about the retention of molars after root-resection therapy was done. They conclude that the prognosis of root resection is well documented in the literature, but remarkable heterogeneity is noticeable when comparing different studies. ${ }^{18}$

The authors conclude that: 18

- The cumulative survival probability decreased from 98.9\% (5 years) to 90.6\% (10 years), 68.9\% (15 years), $43.6 \%$ (20 years) and $34.9 \%$ after 25 to 30 years.

- Lower molars showed a survival probability of almost 80\% even 20 years after root resection.

- Upper molars were lost more frequently than lower molars.

- There is not an indication for tooth extraction of upper and lower molars, but almost all maxillary molars were lost after the resection of the palatal root.

- Most of the teeth extractions reported after root resection in different studies are caused by reasons other than periodontal disease recurrence.

- The main reasons for extraction are endodontic-related 
complications, caries, and restorative problems.

The high survival rates of single crown implants (97.7\%; 94.9\%) and implant-supported fixed dental prostheses (93.6\%; 86.7\%) at 5 and 10 years are attractive to be considered as an alternative to periodontal treatment, the extraction of the periodontal compromised teeth and the posterior rehabilitation with a dental implant. ${ }^{19}$

In case of a maxillary molar the problem is that advanced furcation lesion induces a substantial reduction of the alveolar bone height is observed so, in case of extraction, the amount of remaining bone is further reduced due to vertical ridge resorption and consequently the implant placement is compromised or requires additional regeneration techniques like sinus floor elevation or requires the use of short implants. ${ }^{20,21}$

Another issue to take in account when we decide the most favourable treatment is that the survival rates of implants in the posterior maxilla are less favourable and implants placed in patients who were treated for periodontal disease, are associated with a higher incidence of biological complications. ${ }^{20,22}$

In the study of Derks et al, the survival of resected molars after 10 years was $90.6 \%$, which is not significantly different from the survival implants rate. ${ }^{18}$ In another study Fugazzotto et al, reported a 15-year cumulative success rate of $96.8 \%$ for molars that have been submitted to a root-resected and $97 \%$ for implant placed in the molar area. ${ }^{23}$

\section{CONCLUSIONS}

The literature reports different management techniques to treat furcation lesions. All of them have different treatment indication, but performed following the indications, all of them present excellent results in the long term.

Similar survival rates are described in the literature when we compared the root separation and resection with the dental implants survival rate, but the first one is cheaper and allows to keep the natural tooth.

This clinical case shows that, with a highly motivated patient and with the complete periodontal treatment, is possible to obtain excellent results with root separation and resection in maxillary molars class III.

\section{CONFLICT OF INTEREST}

The authors declares that there is no conflict of interest regarding the publication of this article.

\section{REFERENCES}

1. Schou S, Holmstrup P, Worthington HV, Esposito M. Outcome of implant therapy in patients with previous tooth loss due to periodontitis. Clin Oral Implants Res. 2006;17 Suppl 2:104-23.

2. Carranza FA, Jr., Jolkovsky DL. Current status of periodontal therapy for furcation involvements. Dent Clin North Am. 1991;35(3):555-70.

3. Carnevale G, Pontoriero R, di Febo G. Long-term effects of root-resective therapy in furcation-involved molars. A 10-year longitudinal study. J Clin Periodontol. 1998;25(3):209-14.

4. Lindhe J, Lang NP, Karring T. Clinical Periodontology and Implant Dentistry. $5^{\circ}$ ed. Publishing B, editor. Oxford: $2008 ; 2008$.

5. Hamp S-E, Nyman S, Lindhe J. Periodontal treatment of multi rooted teeth.. Results after 5 years. Journal of Clinical Periodontology. 1975;2(3):12635.

6. Lindhe J, Hamp SE, Loe H. Plaque induced periodontal disease in beagle dogs. A 4-year clinical, roentgenographical and histometrical study. J Periodontal Res. 1975;10(5):243-55.

7. Armitage GC. Development of a classification system for periodontal diseases and conditions. Ann Periodontol. 1999;4(1):1-6.

8. Petersen PE, Ogawa H. Strengthening the prevention of periodontal disease: the WHO approach. J Periodontol. 2005;76(12):2187-93.

9. Heitz-Mayfield LJ, Trombelli L, Heitz F, Needleman I, Moles D. A systematic review of the effect of surgical debridement vs non-surgical debridement for the treatment of chronic periodontitis. J Clin Periodontol. 2002;29 Suppl 3:92-102; discussion 60-2.

10. C L, M P, RF A. Lesão de Furca: Tratamento Periodontal versus Colocação de Implantes. Rev Port Estomatol Cir Maxilofac. 2006;47:117-25.

11. Pjetursson BE, Asgeirsson AG, Zwahlen M, Sailer I. Improvements in implant dentistry over the last decade: comparison of survival and complication rates in older and newer publications. Int J Oral Maxillofac Implants. 2014;29 Suppl:308-24.

12. Pretzl B, Wiedemann D, Cosgarea R, Kaltschmitt J, Kim TS, Staehle HJ, et al. Effort and costs of tooth preservation in supportive periodontal treatment in a German population. J Clin Periodontol. 2009;36(8):669-76.

13. Cortellini P, Buti J, Pini Prato G, Tonetti MS. Periodontal regeneration compared with access flap surgery in human intra-bony defects 20 -year follow-up of a randomized clinical trial: tooth retention, periodontitis recurrence and costs. J Clin Periodontol. 2017;44(1):58-66.

14. Halperin-Sternfeld M, Levin L. Do we really know how to evaluate tooth prognosis? A systematic review and suggested approach. Quintessence Int. 2013;44(5):447-56.

15. Kwok V, Caton JG. Commentary: prognosis revisited: a system for assigning periodontal prognosis. J Periodontol. 2007;78(11):2063-71.

16. McGuire MK, Nunn ME. Prognosis versus actual outcome. II. The effectiveness of clinical parameters in developing an accurate prognosis. J Periodontol. 1996;67(7):658-65.

17. Livada R Fau - Fine N, Fine N Fau - Shiloah J, Shiloah J. Root amputation: a new look into an old procedure. N Y State Dent J. 2014;80(4):24-8.

18. Derks H, Westheide D, Pfefferle T, Eickholz P, Dannewitz B. Retention of molars after root-resective therapy: a retrospective evaluation of up to 30 years. Clinical oral investigations. 2017.

19. Albrektsson T, Donos N, Working G. Implant survival and complications. The Third EAO consensus conference 2012. Clin Oral Implants Res. 2012;23 Suppl 6(1600-0501 (Electronic)):63-5.

20. Walter C, Dagassan-Berndt DC, Kuhl S, Weiger R, Lang NP, Zitzmann NU. Is furcation involvement in maxillary molars a predictor for subsequent bone augmentation prior to implant placement? A pilot study. Clin Oral Implants Res. 2014;25(12):1352-8.

21. MacBeth N, Trullenque-Eriksson A, Donos N, Mardas N. Hard and soft tissue changes following alveolar ridge preservation: a systematic review. Clin Oral Implants Res. 2017;28(8):982-1004.

22. Sousa V, Mardas N, Farias B, Petrie A, Needleman I, Spratt D, et al. A systematic review of implant outcomes in treated periodontitis patients. Clin Oral Implants Res. 2016;27(7):787-844.

23. Fugazzotto P, Melnick PR, Al-Sabbagh M. Complications when augmenting the posterior maxilla. Dent Clin North Am. 2015;59(1):97-130. 